\title{
Performance Evaluation of Frame Relay, SMDS, and ABR Services in ATM Networks
}

\author{
L. Fratta, L. Musumeci \\ Dipartimento di Elettronica e Informazione, Politecnico di Milano \\ Piazza Leonardo da Vinci 32, I-20133 Milano, Italy \\ tel. $+39223993578-f a x+39223993413-$ \\ e-mail:fratta@elet.polimi.it
}

\begin{abstract}
The broadband integrated services digital networks are based on the ATM technology. As these networks will be deployed gradually, the existing high-speed data services, as Frame Relay and SMDS, will continue to exist and, for interoperability purposes, they will also be supported by the ATM networks. Moreover, ATM networks allows to efficiently support a new service, called available bit rate (ABR) service in the ATM Forum, which requires no bandwidth reservation. When this service is requested, data are transmitted through the network with low priority, being the available network resources shaped dynamically among all the active sources. In this paper, we first discuss how to manage FR and SMDS services in the ATM networks, and report the performance evaluation of the proposed interconnection scenarios; then, we present some preliminary simulation results on the ABR service, whose implementation needs a sophisticated reactive congestion control to obtain an acceptable quality of service.
\end{abstract}

\section{Keywords}

Frame Delay, SMDS, ABR, ATM network, rate-based control.

\section{INTRODUCTION}

The increasing demand for high-speed data switched services has stimulated the introduction of new "fast packet" technologies. In particular, Frame Relay (FR) has appeared appropriate to support new data applications, such as LAN-to-LAN interconnection at $2 \mathrm{Mbit} / \mathrm{s}$. Frame relay [1] is a connection-oriented technology that supports variable-length packets and is designed to improve the efficiency of X.25 packet networks.

In addition, SMDS (Switched Multimegabit Data Service) represents another strategy to meet the demand for high-speed data services up to $45 \mathrm{Mbi} / \mathrm{s}$ before the introduction of the 
broadband integrated services digital networks, B-ISDN. The SMDS interface and associated protocols are based on the connectionless part of the IEEE 802.6 distributed queue dual bus (DQDB) MAN standard [1].

While the scope of FR and SMDS networks is to support only high-speed data applications, the Asynchronous Transfer Mode (ATM) technology has been proposed as a standard for supporting B-ISDN. ATM is based on the concept of a homogeneous network, where all traffic is transformed into a uniform 53-byte packet or cell stream. This allows the network to carry a wide variety of different traffic types [1].

As B-ISDN deployment will be gradual, ATM will not replace Frame Relay and SMDS services in the near future. It is expected that these services will be supported by the ATM networks since the initial phase of their deployment.

The introduction of ATM technology offers new problems and challenges, which have been subject of intense research over the past few years. The ATM Forum has promoted an important work, devoted to accelerate the development and standardization of ATM technology.

Four traffic classes, constant bit rate (CBR), variable bit rate (CVR), available bit rate (ABR), and unspecified bit rate (UBR), have been proposed on the ATM Forum [2]. For CBR and VBR services, the ATM networks guarantee the negotiated quality of service. For example, a CBR service is described in terms of peak cell rate (PCR) and cell delay variation (CDV). For this service, congestion control is managed through admission control and bandwidth allocation procedures. Therefore, if the resources requested are not available, the connection will be rejected at the call setup phase.

For many conventional computer applications, it is not easy to predict the bandwidth requirements. In these cases, it is not required to reserve bandwidth in the network and it is more appropriate to share the available bandwidth among all active users. Such a service, the available bit rate $(\mathrm{ABR})$ service, requires the application of an explicit control scheme to reduce cell losses.

The congestion control for ABR service has been standardized in the ATM Forum's September 1994 meeting. The rate based control for ABR services was preferred to the credit control scheme, which requires extremely large buffers in each switch in the wide-area networks with many VCs and large propagation delays.

The goal of this paper is twofold. First, we discuss some proposals to achieve an efficient management of FR and SMDS services, when supported in an ATM network. In particular, we investigate the impact of FR traffic on the performance characteristics of ATM networks and discuss how to improve the cell loss probability and the fairness due to the presence of the FR traffic in the ATM multiplexer. In addition, we describe the access of the SMDS traffic to the ATM network through a Two Rate Multiplexer (TRM), which allows to dynamically manage the bandwidth of a Virtual Path (VP) towards a Connectionless Server (CLS) through an ATM switch. A model of this TRM is presented together with its performance evaluation. Second, we present our preliminary simulation results on the ATM Forum proposal for the ABR service illustrating its performance characteristics. Finally, we conclude with some considerations on the future extension of this work.

\section{FRAME RELAY SERVICE IN ATM NETWORKS}

The LAN interconnection based on FR interfaces, can be implemented in an ATM network by the use of dedicated Virtual Paths (VP), dimensioned at the peak bit rate. This approach is 
simple, but not efficient. More efficient solutions can be obtained by statistically multiplexing the FR traffic with ATM sources. To investigate the impact of FR traffic on ATM multiplexer performance, we consider the network model of figure 1, where the FR multiplexer is colocated with the ATM multiplexer. The FR sources are connected to the FR multiplexer via 2 Mbps links. Each FR source can adjust its current window size between a minimum $(W=1)$ and a maximum $(\mathrm{W}=7)$ value, according to network congestion status. The generated frames, which can not be forwarded, are stored in the source buffer. The FECN/BECN mechanism, implemented in the FR multiplexer, allows to notify the network congestion to the FR sources. In the FR multiplexer, a transmitted frame has the FECN bit set to one, if the multiplexer is congested. Each transmitted frame is acknowledged by the destination through an ACK frame. This frame is addressed to the source with the BECN bit set to one, if the corresponding frame was received with the FECN bit set to one. An ACK frame is received by the source after an overall round-trip delay $\tau$. In presence of network congestion, each source reduces his window to a value $r W$, where $r$ is a preassigned reduction factor. Otherwise, the window is increased by one up to the maximum value. The algorithm to measure the network congestion in the FR multiplexer and the algorithm to modify the window in each source are described in more details in [3]. Each FR frame is segmented into 32 ATM cells before accessing the ATM multiplexer.

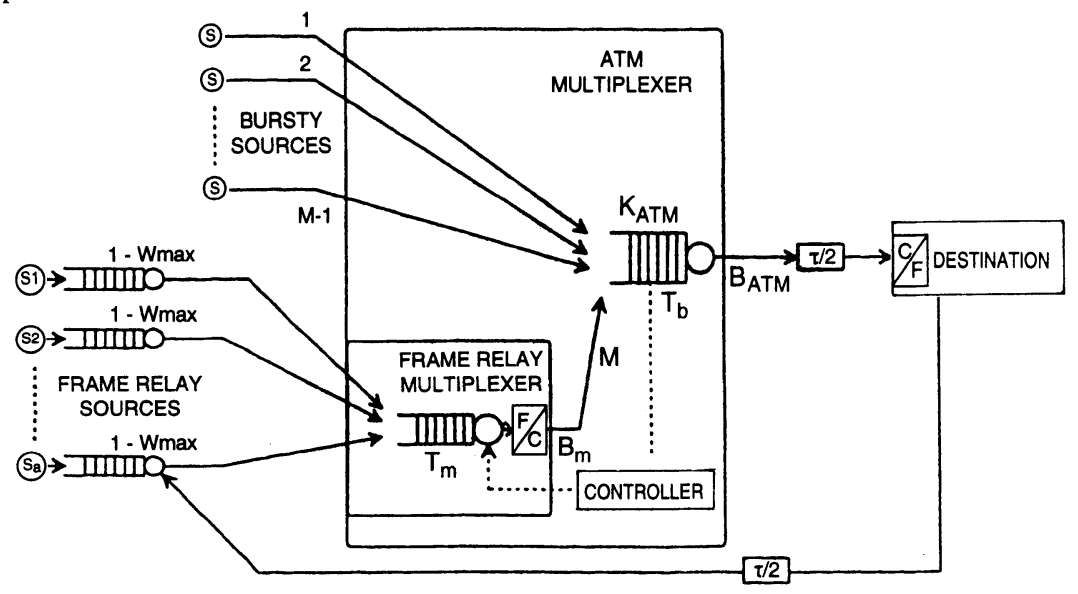

Figure 1 Architecture for the FR access in an ATM multiplexer.

$\mathrm{M}=10$ bursty sources are connected to an ATM multiplexer, which is characterized by a single server with a finite buffer $\mathrm{K}_{\mathrm{ATM}}$. The ATM multiplexer has a buffer capacity $\mathrm{K}_{\mathrm{ATM}}=50$ cells and a transmission bit rate $B_{A T M}=150$ Mbps. Each source generates traffic with peak bit rate $B_{p}=34 \mathrm{Mbps}$, mean burst length $L_{m}=100$ cells and burstiness $b_{b}$, which is selected in order to obtain a proper average load on each access link, $\rho_{b}$. The simulation allows to evaluate the cell loss probability $\mathrm{P}_{\mathrm{e}}$ as function of $\rho_{\mathrm{b}}$.

To investigate the effect of the FR traffic on the ATM multiplexer cell loss probability, one ATM bursty source has been substituted by a 34 Mbps FR multiplexer. We suppose that FECN and BECN bits are also supported by the ATM cell header. Therefore, in our model, both FR and ATM multiplexers can provide explicit congestion information, at the frame and at cell level, respectively, when congestion is detected in the corresponding buffer. 
Simulation results confirm that the overall cell loss probability remains the same as in the case of only bursty sources connected to the ATM multiplexer, if the average load of the FR traffic, $\rho_{F R}$, is equal to $\rho_{\mathrm{b}}$. As the average load, $\rho_{\mathrm{FR}}$, of the FR multiplexer increases, the cell loss probability also increases, as shown in figure 2 .

In order to discuss the cell loss probability fairness, in Table 1 we have reported the simulation results obtained with $\rho_{F R}=0.4$, which corresponds to a FR traffic load equal to $43 \%$ of the total traffic offered to the ATM multiplexer. In these conditions, the overall cell loss probability is less than $10^{-4}$.

ATM bursty sources suffer a worse cell loss probability, compared to the FR traffic. This behavior is a consequence of the fact that the FR sources transmit at 2 Mbps and are multiplexed at $34 \mathrm{Mbps}$. The better performance of FR traffic is obtained at the cost of a cell loss probability, suffered by bursty sources, which is higher than the ATM multiplexer overall cell loss probability. This unfairness penalizes the bursty sources in favor of the FR traffic. Alternative solutions have been investigated [3] in order to reduce the drawback caused by the presence of the FR traffic.

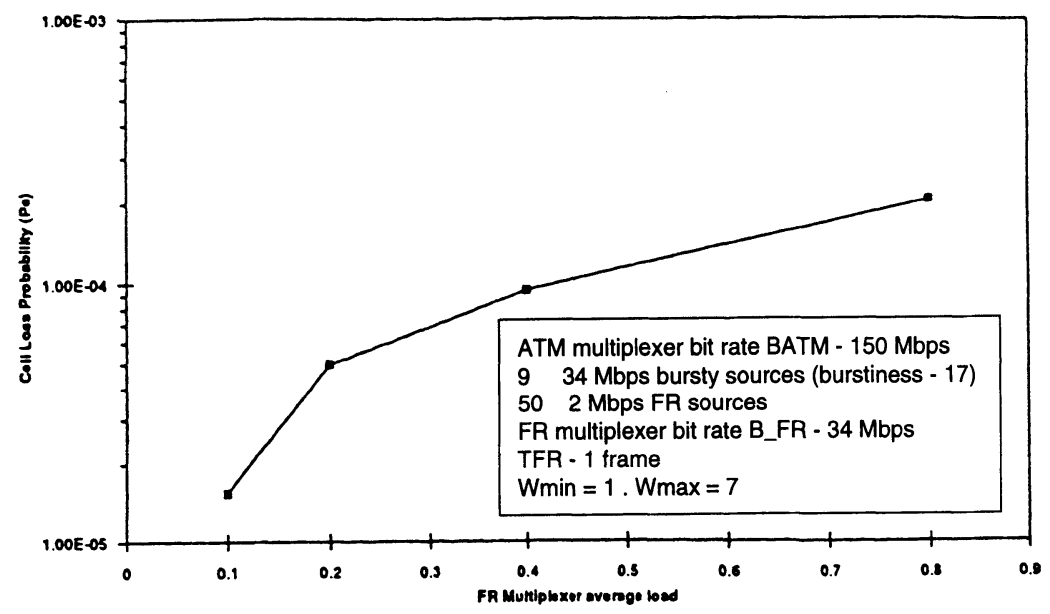

Figure 2 ATM cell loss probability as a function of the FR average load.

Table 1 Simulation results on FR fairness and delay in the basic scheme.

Overall ATM Cell Loss Probability $9.00 \mathrm{E}-05$

Frame Relay Cell Loss Probability 1.50E-05

Bursty sources Cell Loss Probability $\quad 1.46 \mathrm{E}-04$

FR Mean File Delay [s]

SR File Delay Standard Deviation [s] 
A first alternative is to introduce a Burst Length Shaping function (BLS) [4] in the FR multiplexed traffic. The shaping function permits to limit the FR cell burst length to a maximum value $L_{\max }$. Whenever a maximum-length burst occurs, an appropriate silent time is inserted [4]. By reducing the maximum length, $L_{\max }$, of the cell bursts, the mean length of the FR bursts increases, as long as $\mathrm{L}_{\max }$ is greater than the mean length of the bursts resulting from multiplexing FR sources. Therefore, FR and bursty traffic characteristics become more similar and cell loss unfairness tends to disappear, as shown in Table 2. The main drawback of this mechanism is the increase in the FR end-to-end file transmission delay, as resulting from the comparison between the values of Table 1 and Table 2.

Table 2 Comparison of simulation results on FR fairness and delay.

\begin{tabular}{|c|c|c|c|c|}
\hline & $\begin{array}{c}\text { BLS } \\
\left(\mathrm{L}_{\max }=1200\right)\end{array}$ & $\begin{array}{c}\text { BLS } \\
\left(\mathrm{L}_{\max }=300\right)\end{array}$ & IFRC & BFRC \\
\hline $\begin{array}{l}\text { Overall ATM Cell Loss } \\
\text { Probability }\end{array}$ & $7.00 \mathrm{E}-05$ & $5.00 \mathrm{E}-05$ & 4.00E-06 & $1.00 \mathrm{E}-06$ \\
\hline $\begin{array}{l}\text { Frame Relay Cell Loss } \\
\text { Probability }\end{array}$ & $8.00 \mathrm{E}-06$ & $6.20 \mathrm{E}-05$ & $<1 \mathrm{E}-07$ & $<1 \mathrm{E}-07$ \\
\hline $\begin{array}{l}\text { Bursty Sources Cell Loss } \\
\text { Probability }\end{array}$ & $1.10 \mathrm{E}-04$ & 4.20E-05 & $6.00 \mathrm{E}-06$ & $1.35 \mathrm{E}-06$ \\
\hline FR Mean File Delay $[\mathrm{s}]$ & 0.973 & 1.522 & 0.879 & 0.845 \\
\hline $\begin{array}{l}\text { FR File Delay Standard } \\
\text { deviation }[\mathrm{s}]\end{array}$ & 1.01 & 1.498 & 1 & 0.97 \\
\hline
\end{tabular}

The second method, integrated FR Control (IFRC), introduces a control mechanism on the FR multiplexer.

When the ATM buffer occupancy reaches a given threshold value $T_{b}$, the FR server is disabled and no FR frame can access the ATM buffer. The FR server is enabled when the ATM buffer occupancy drops below $T_{b}$. This method, implemented with a threshold value $\mathrm{T}_{\mathrm{b}}=1$ cell, produces a dramatic decrease of the cell loss probability in the ATM multiplexer for both FR and bursty sources, even if the fairness is not improved. The reduction of the overall cell loss probability is due to the fact that the FR traffic enters the ATM buffer only when the total load is low (queue length smaller than $T_{b}$ ). This way of operation transfers network congestion situations to the FR multiplexer, which has the means, based on the ECN mechanism, to reduce the source FR traffic. The additional delay incurred by the FR traffic is negligible, as shown in Table 2.

The integrated control can be implemented only if the access ATM multiplexer includes the FR multiplexing stage. If the two multiplexers are remote, a similar method, the Buffer-based FR Control (BFRC), can be implemented by storing the FR cells in an additional FR-dedicated ATM buffer, when the access to the buffer $\left(\mathrm{K}_{\mathrm{ATM}}\right)$ is forbidden. This implementation further enhances multiplexing performance in terms of cell loss probability and transmission delay, because FR traffic control is performed on a per-cell basis, and not on a per-frame basis as in the previous case. 
The best performance is provided by the BFRC, which reduces the overall cell loss probabilities of two orders of magnitude. This improvement is obtained by preventing the FR traffic to enter the ATM buffer when it is not empty. The additional delay due to this operation has no effect on the FR file delay.

\section{SMDS ACCESS IN ATM NETWORKS}

As ATM networks offer connection-oriented services, the provision of connectionless data services requires that each Interworking Unit, which interfaces the users, is interconnected to the ATM network by a permanent Virtual Path (VP) or a permanent Virtual Channel (VC). In this strategy, the ATM network is completely transparent to the connectionless service. This solution is very simple, but its efficiency is very limited if the IWU operates at the peak bit rate, even if the connectionless traffic is bursty. Moreover, the use of permanent connections between all pairs of IWUs is feasible only for small size networks. A more efficient strategy makes use of Connectionless Servers (CLS) in ATM network, as proposed by ITU [5]. In this approach, each IWU may be connected to a CLS through a VP, whose bandwidth can be permanently or dynamically allocated. A Variable Rate Multiplexer (VRM), which selects its output transmission speed, $\mathrm{V}_{\mathrm{o}}$, in relation to the traffic load and the buffer occupancy, presents a high effectiveness.

We proposed a simple implementation of a VRM, obtained with the use of only two values of the output speed $\mathrm{V}_{\mathrm{o}}[6]$. The two rate multiplexer, TRM, is described by the following three parameters:

- the nominal bandwidth, $\mathrm{V}_{\mathrm{N}}$;

- the maximum bandwidth, $\mathrm{V}_{\mathrm{M}}$;

- the utilization factor, $\mathrm{F}$, of $\mathrm{V}_{\mathrm{M}}$.

The bandwidth $V_{N}$ is permanently allocated in the ATM network, while $V_{M}$ is used, when needed, for limited periods of time. Its average use is given by $F$.

The performance of the TRM has been obtained through a PTS (PDU-Transmission Scheduling) algorithm, whose goal is to monitor the traffic and to verify if the lower output speed can be used without violating the declared frame loss probability.

As specified in SMDS, the L3-PDU frames are segmented in cells. The first one, BOM, specifies the length of the frame and the last one, EOM, specifies the end of the frame. At the reception of a BOM in the TRM, the PTS algorithm evaluates which output speed, $V_{N}$ or $V_{M}$, permits to maintain the buffer occupancy, $Q$, at the minimum value below the permitted threshold $\left(Q_{\max }\right)$ for all frame being transmitted. If this condition can not be satisfied, the new incoming frame is rejected and the transmission speed remains unchanged. Moreover, at the reception of an EOM, a decision is taken whether to decrease the transmission speed. Details of this algorithm were presented in [6].

The parameter $V_{N}$ highly influences the performance characteristics, as shown in figures 3 , 4 , and 5, that summarize the simulation results observed for different values of $Q$ and assuming $\mathrm{N}$ sources transmitting at $\mathrm{V}_{\mathrm{I}}=5 \mathrm{Mbps}$ with an activity $\mathrm{A}=0.5 \mathrm{Mbps}$. The frame length is geometrically distributed with average length $\mathrm{L}=5$. The frame rejection probability, $\mathrm{p}_{\mathrm{R}}$, (figure 3 ) presents a maximum, as $V_{N}$ changes, which also depends on the buffer length. To reduce $p_{R}$ for a given offered traffic, either a very high value, close to $V_{M}$, or a small value, close to the average offered traffic, should be chosen. 


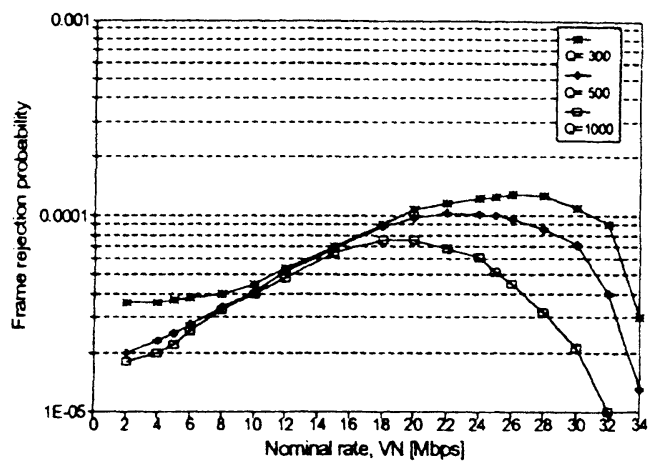

Figure 3 Frame rejection probability versus the nominal rate in the TRM for different values of Parameter $Q$

$\left[\mathrm{V}_{\mathrm{I}}=5 \mathrm{Mbps}, \mathrm{A}=0.5 \mathrm{Mbps}, \mathrm{N}=18, \mathrm{~L}=5\right.$ frames].

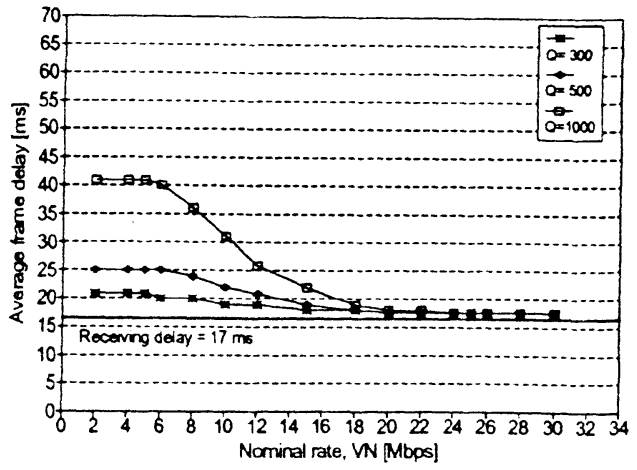

Figure 4 Average frame delay versus the nominal rate in the TRM for different values of parameter $Q$

$\left[\mathrm{V}_{\mathrm{I}}=5 \mathrm{Mbps}, \mathrm{A}=0.5 \mathrm{Mbps}, \mathrm{N}=18\right.$, $\mathrm{L}=5$ frames].

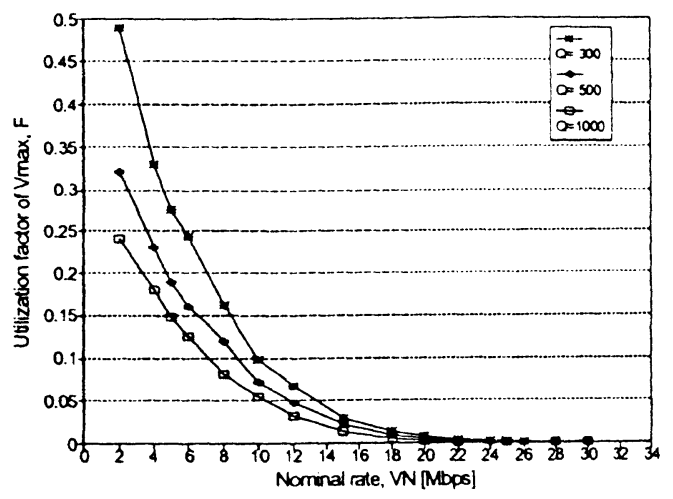

Figure 5 Utilization factor of $V_{M}$ versus the nominal rate $\left[V_{I}=5 \mathrm{Mbps}, A=0.5 \mathrm{Mbps}\right.$, $\mathrm{N}=18, \mathrm{~L}=5$ frames $]$.

The frame delay, $\tau$, changes with $V_{N}$ only for values of $Q_{\max }$ greater or equal to the average source burst size (figure 4). The utilization factor $F$ is very high for small values of $V_{N}$ and rapidly decreases as $\mathrm{V}_{\mathrm{N}}$ increases (figure 5).

For a required $p_{R}$, two sets of values for $V_{N},\left\{V_{N}<V_{N 1}\right\}$ and $\left\{V_{N}>V_{N 2}\right\}$, are possible according to the results shown in figure 3 . The corresponding values of the delay and the utilization factor are given in figures 4 and 5 , respectively. For a given $V_{N}$, several choices of the buffer size are possible to satisfy the constraints given in terms of $p_{R}, \tau$, and $F$. The best choice of $V_{N}$ is obtained by minimizing the "Equivalent Bandwidth", $B E\left(V_{N}\right)$, defined as: 
$\mathrm{BE}\left(\mathrm{V}_{\mathrm{N}}\right)=\left[1-\mathrm{F}\left(\mathrm{V}_{\mathrm{N}}\right)\right] * \mathrm{~V}_{\mathrm{N}}+\mathrm{F}\left(\mathrm{V}_{\mathrm{N}}\right) * \mathrm{~V}_{\mathrm{M}}$

To keep the bandwidth allocation as small as possible, it is more appropriate to find solutions characterized by values of $V_{N}$ about equal to the average offered traffic.

Table 3 Comparison among VRM, TRM, SM, and PM [ $\mathrm{V}_{\mathrm{I}}=5 \mathrm{Mbps}, \mathrm{A}=0.5 \mathrm{Mbps}, \mathrm{L}=5$ frames, $Q_{\max }=1000$ cells, $p_{R}<510^{-5}$.

\begin{tabular}{lcccc}
\cline { 2 - 4 } Vo [Mbps] & VRM & TRM & SM & PM \\
N & $5,10,15, \ldots, 34$ & 10,34 & 34 & 34 \\
N A [Mbps] & 12 & 18 & 23 & 7 \\
F & 6 & 9 & 11.5 & 3.5 \\
BE [Mbps] & --- & 0.06 & 1 & 1 \\
E & 6 & 11.4 & 34 & 34 \\
\hline
\end{tabular}

In Table 3, some numerical results permit to compare the TRM with the Statistical Multiplexer (SM) and the Peak Multiplexer (PM), which assigns the peak bandwidth to each source. To compare the different schemes, we have defined the efficiency, $\mathrm{E}$, as the ratio between the equivalent bandwidth and the total average throughput. A reference multiplexer, called Variable Rate Multiplexer (VRM), that can adjust the output speed $V_{o}$ within a set of values, is also taken into account. Its efficiency is equal to 1 . In the PM, the efficiency E equals the source burstiness. Both the SM and the TRM schemes present an intermediate behavior. The higher multiplexing gain provided by SM is obtained at the cost of a very low efficiency $(\mathrm{E}=$ $2.95)$. On the contrary, the efficiency $(E=1.26)$ obtained by TRM is only $26 \%$ worse than the optimum value. The PM is the simplest multiplexer, but it provides too poor performance. TRM and SM present the same level of complexity, as both require a policing mechanism.

The effective use of the TRM requires the control of its parameters in order to guarantee the ATM network the declared traffic characteristics. While the control of $V_{N}$ is relatively simple, the control of $\mathrm{F}$ presents some problems. In fact, the utilization factor, theoretically defined on an infinite time, must be measured and controlled on a finite time interval. Furthermore, such an interval must be short enough to guarantee that cell bursts, transmitted at $V_{M}$ by the TRM, are shorter than a given value $\mathrm{T}_{\mathrm{M}}$ declared to the ATM network.

In [6], a "Time-Credit" mechanism has been proposed, based on a time-window of fixed length $\mathrm{W}$. According to this mechanism, the TRM is allowed to transmit at $\mathrm{V}_{\mathrm{M}}$ within $\mathrm{W}$ for a total time up to $\mathrm{F} * \mathrm{~W}$. Once the Time Credit $\mathrm{F} * \mathrm{~W}$ has been spent, only the transmission speed $\mathrm{V}_{\mathrm{N}}$ can be used for the remaining time. The performance of the Time-Credit mechanism is shown in figure 6, where the frame rejection probability, $\mathrm{p}_{R}$, is given as function of $\mathrm{W}$ for different values of $F$. For a given $F, p_{R}$ increases as $W$ decreases. As a consequence, to guarantee low values of $p_{R}$ with small value of the window, it necessary to use high value of the utilization factor, that is, in other words, to work at the peak bit rate. 


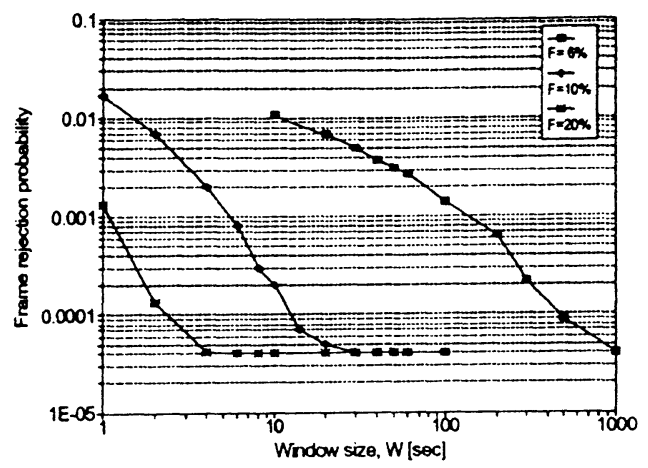

Figure 6 Frame rejection probability vs the window size in the TRM for different value of parameter $F\left[V_{I}=5\right.$ Mbps, $A=0.5 \mathrm{Mbps}, \mathrm{N}=18, \mathrm{~L}=5$ frames, $\mathrm{V}_{\mathrm{N}}=10 \mathrm{Mbps}, \mathrm{Q}=1000$ cells].

\section{ABR SERVICES IN ATM NETWORKS}

Initially, CBR services, described in terms of peak bit rate and cell delay variation, will be provided by ATM networks. In addition, ABR services are also supported in an ATM environment for conventional computer communications, such as in the current TCP/IP, where no bandwidth reservation is required. As cells may be lost during the congestion period due to the buffer overflow in the ATM switches, it is necessary, even if large buffers are considered, to use reactive congestion control to prevent such cell losses in ABR services.

According to the ATM Forum indication, we only consider the rate-based control, which is very appropriate to work both in LAN and WAN scenarios. Such kind of control requires only the definition of the end-system behavior and leaves flexibility in both the design of the switch architecture and in the selection of traffic management strategy [7].

\subsection{ATM Forum Rate-based Control}

The rate-based control, implemented using traditional FECN and BECN schemes [8], presents an unsafe behavior as notification cells can be delayed or lost when a severe congestion is experienced in the network. In fact, if no congestion notification is received, the source continues to increase its transmission rate at regular time intervals up to the allowed peak value.

To overcome this serious drawback, the ATM Forum has proposed a proportional rate control algorithm (PCRA), where a source increases its transmission cell rate only at the reception of an explicit notification, indicating no congestion in the network [7, 8]. Otherwise, the source gradually reduces its cell rate until the minimum allowed value is reached.

In the following, we describe an enhanced version of the PRCA, E-PRCA, reported in [9], but modified in some parts to simplify the functions requested at the ATM switches.

The operation of E-PRCA is based on the information carried by the resource management (RM) cells. Before the transmission begins, the source sets the allowed cell rate (ACR) to the initial cell rate (ICR) value, negotiated during the virtual connection setup. Then, the data 
transmission phase, consisting of a sequence of one RM cell followed by $\mathrm{N}$ data cells, can start. The information carried by the RM cells includes:

- DIR, RM cell direction (forward/backward);

- CCR, current source cell rate;

- MCR, minimum source cell rate, chosen at the connection setup;

- CI, congestion indication;

- ER, explicit cell rate.

CCR and MCR are set by the source, DIR by both source and destination, while CI and ER are set by source and network switches. ER is initially set equal to the peak cell rate (PCR) by the source and possibly reduced by any node on the $\mathrm{VC}$, which has an available bandwidth smaller than the current value of ER. The CI flag is set to " 0 " by the source and changed to "1" by any congested switch.

The data cell transmission rate is decreased at each RM cell, i. e. every $\mathrm{N}$ cells, according to the relation:

\section{$\mathrm{ACR}=\mathrm{ACR} *[1-(1 / \mathrm{RDF})]$}

where the value of RDF (Rate Decrease Factor) is negotiated at connection set up. The data cell rate is increased at the reception of an RM cell carrying no congestion information, of a value equal to the last decrease value plus an amount, AIR (Additional Increase Rate), negotiated at the connection set up.

\subsection{Reference Network Model}

In order to investigate the E-PRCA performance, we have simulated a simple network model shown in figure 7 .

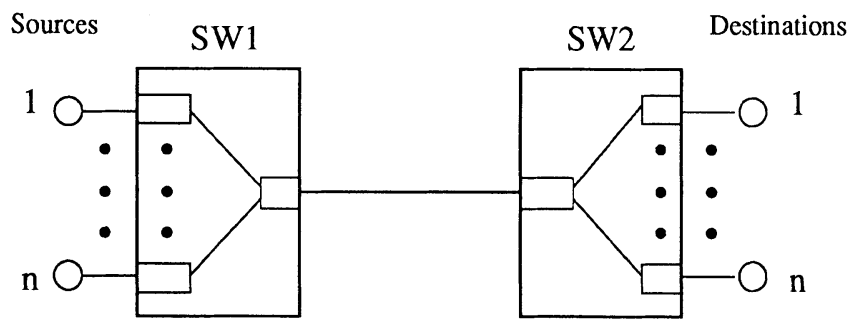

Figure 7 Reference network model for E-PRCA performance evaluation.

Traffic sources are attached to switch 1, while destinations are attached to switch 2. Each source has one virtual channel (VC) for a unique destination. Distance D1 in the access loop, and distance D2 between the two switches can be varied to capture the effect of the propagation delay on the control performance. Distance D1 may be different for different sources. 
Each switch is modeled as a buffered switch with buffers at each input and output port [10]. Internally, cells are forwarded from input buffer to output buffer according to a random in random out scheduling to avoid unfairness. A backpressure policy is enforced between the output and input buffers to avoid cell loss at output buffers.

Preliminary simulation results have been obtained assuming $\mathrm{MCR}=0.1, \mathrm{PCR}=1, \mathrm{ICR}=0.1$, and $\mathrm{AIR}=0.01$. These values are normalized to the channel rate. Furthermore, we have considered $\mathrm{N}=32$ and $\mathrm{RDF}=128$, as widely used in the literature.

In all our simulations, persistent sources, i.e. sources transmitting at $\mathrm{ACR}$, have been assumed in order to stress the control capability.

The parameter ER has been ignored. Only CI is used to notify congestion. Two policies have been considered to recognize a congested switch. In the first one, the queue length policy, QLP, the congestion is detected when the queue length at an input buffer exceeds a preassigned threshold $T_{2}$. The congestion terminates when all queue lengths become smaller than a preassigned threshold $\mathrm{T}_{1}\left(\mathrm{~T}_{1}<\mathrm{T}_{2}\right)$.

In the second, the length gradient policy, LGP, the congestion is detected taking into account also the gradient of the queue lengths [11]. This mechanism allows to react faster to a suddenly emerging congestion.

Both alternatives have been simulated and the results will be discussed and compared in the next section.

\subsection{Numerical results}

The main goal of our simulation is to investigate the performance of the different alternatives discussed in the previous paragraphs and to evaluate the impact of various parameters. To compare the effectiveness of the two strategies for congestion detection we consider the network reference model of figure 7 with three homogeneous sources, which are activated at different times.

All sources have the same distance from the switch $(10 \mathrm{~km})$ and their transmission rate can reach the maximum value $(155 \mathrm{Mb} / \mathrm{s})$. Figure 8 and 10 show the normalized transmission rate permitted to each source versus the observation time. If only source $A$ is active, the transmission rate grows up to the channel speed. As soon as source B is activated, the channel capacity is equally splitted among the two sources. However, strong variations on the transmission rate are observed. This is due to the delay to recognize the congestion situation in the switch and to activate the rate control in the source A. A similar hehavior is observed, when source $\mathrm{C}$ is activated.

The improvement obtained by using the gradient policy reduces the variation in the transmission rate as shown in figure 10 .

The queue length behavior, shown in figure 9 and 11, reflects the same characteristics in terms of variations. Also regarding this performance, the gradient policy is preferable as it reduces the average queue length by a factor of two. This gain allows to significantly reduce the buffer size, even if a large queue length variation has been measured during transient periods, corresponding to the activation of new sources. 


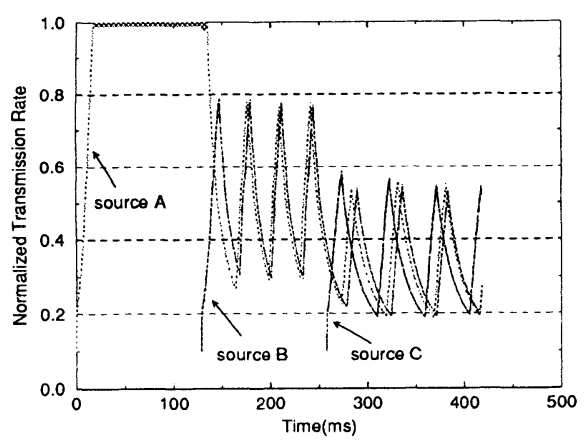

Figure 8 Normalized transmission rate for three homogeneous sources using queue length policy congestion control.

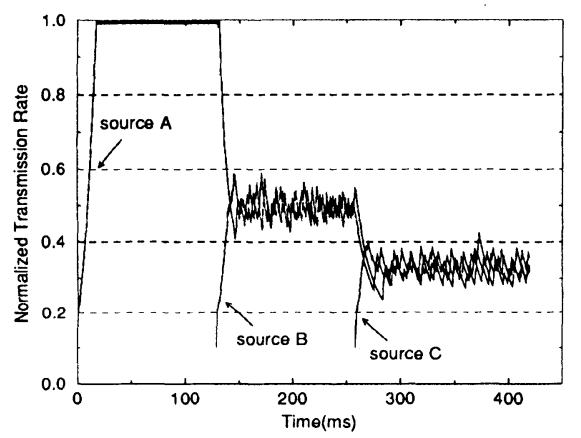

Figure 10 Normalized transmission rate for three homogeneous sources using length gradient policy congestion control.

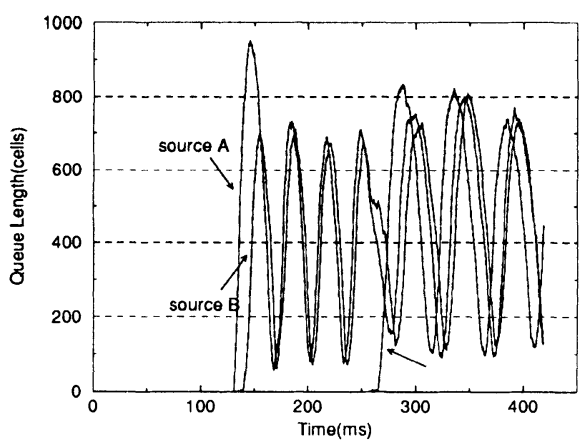

Figure 9 Queue length for three homogeneous sources using queue length policy congestion control.

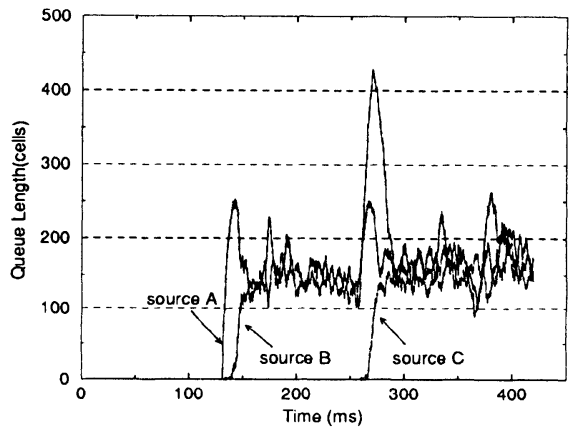

Figure 11 Queue length for three homogeneous sources using length gradient policy congestion control.

A direct comparison between the two techniques, QLP and LGP, is shown in figure 12 and 13 , where only the behavior of source A is reported. In all cases, a fair behavior among the different sources is obtained. 


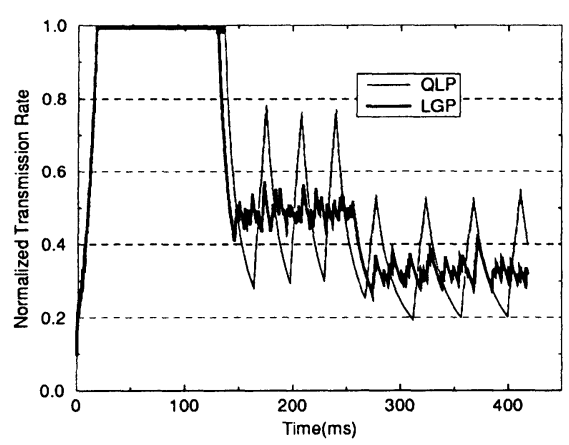

Figure 12 Normalized transmission rate for the source A. A comparison between QLP and LGP.

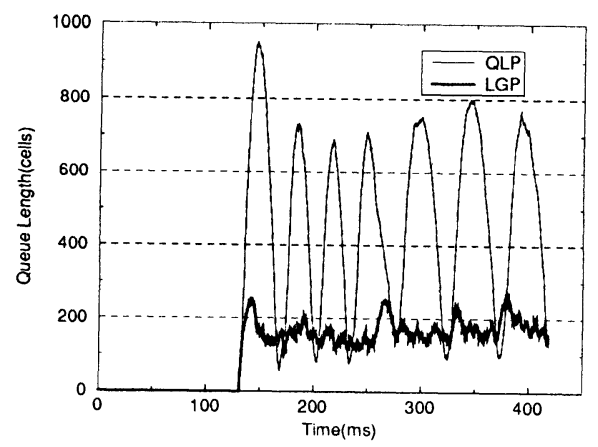

Figure 13 Queue length. A comparison between QLP and LGP.

The choice of ICR has impact on the system performance. High values produce (figure 14) larger variations and therefore larger buffer are necessary.

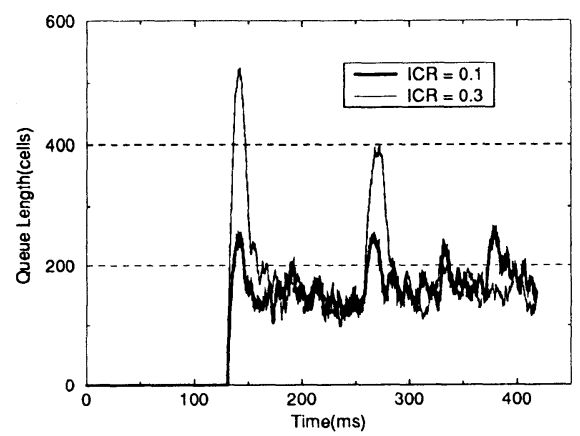

Figure 14 Queue length using GLP. A comparison between ICR $=0.3$ and ICR $=0.1$.

From these preliminary results, it is evident that, no matter which policy is used, the buffers required at the ATM switch are much larger than those considered ( $<100$ cells) in current implementations. The use of small values of ICR is also advisable.

The case of non homogeneous sources has been considered assuming two sources only, one at full channel rate (1) and the other at lower rate (0.3). The ABR service obtained by the faster source is degraded with respect to that of the slower one, which is practically able to work at its nominal bit rate without suffering significant variation in transmission rate and queue length (figures 15,16 ). 


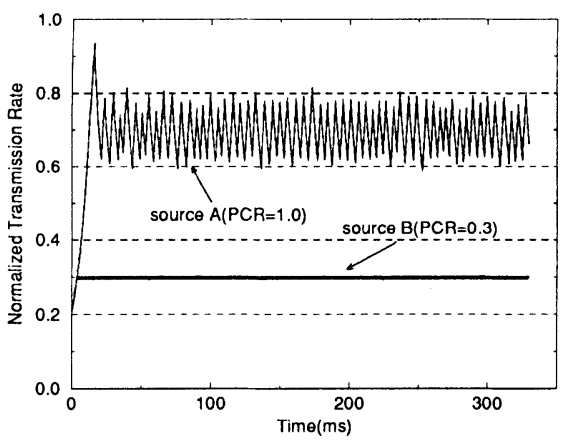

Figure 15 Normalized transmission rate for two non homogeneous sources.

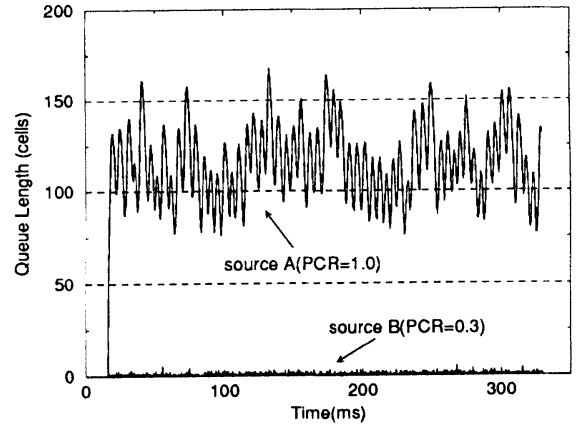

Figure 16 Queue length for two non homogeneous sources.

The effect of the propagation delay is to slow down the source control. This phenomenon has been investigated by assuming two short $(10 \mathrm{~km})$ and a long $(100 \mathrm{~km})$ access links. Figures 17 and 18 show the behavior of normalized transmission rate and queue length, respectively, for a near and the far source. As expected, the far source suffers from more degradation, having higher variations in both transmission rate and queue length. In a multinode network, not considered in this paper, the effect of different propagation delays may be even more critical. To attenuate such a degradation, mechanisms that provide a faster feedback are under investigation.

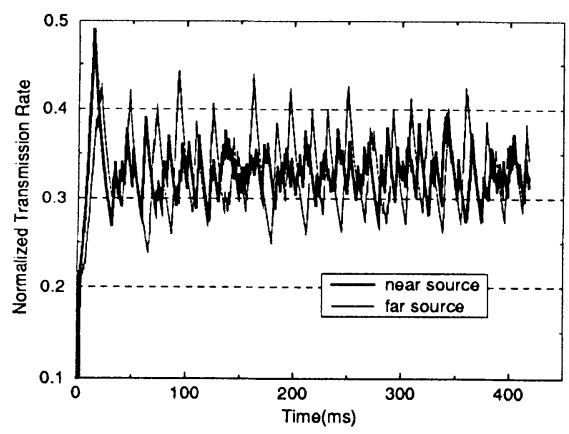

Figure 17 Normalized transmission rate. A comparison between near and far sources.

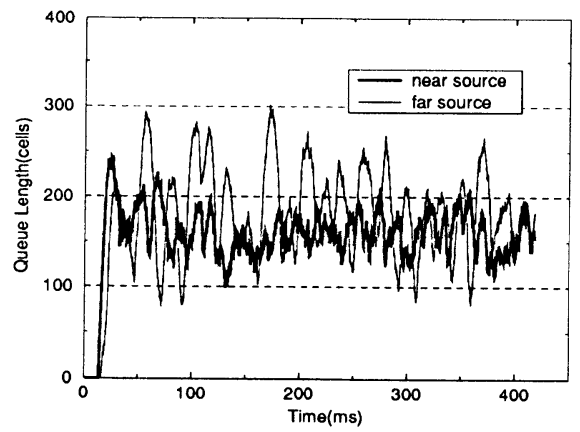

Figure 18 Buffer length. A comparison between near and far sources.

\section{CONCLUSIONS}

Frame Relay and SMDS are fast packet technologies, designed to support high-speed data applications, and, in particular, LAN-to-LAN interconnection. 
As Frame Relay provides a connection-oriented service, it can be implemented on ATM networks based on dedicated virtual path (VP) or, more efficiently, on virtual paths shared with traffic generated by other ATM sources. We have studied this second approach to evaluate the impact of FR traffic on the ATM multiplexer. Simulation results have shown that FR traffic produces an unfair distribution of cell loss probability among different kinds of traffic. To enhance multiplexing performance, different multiplexer structures have been proposed. In particular, the use of additional buffers, dedicated to FR traffic inside the ATM multiplexer, permits to improve the cell loss probability without increasing the end-to-end file transmission delay.

The use of Connectionless Server (CLS), colocated with the ATM switches, is a possible architecture to implement SMDS in an ATM network. In this scenario, each Interworking Unit (IWU) may be connected to a CLS through a permanent VP. Because of the intrinsic bursty characteristic of the offered traffic, the statistical multiplexing operating at the peak bit rate may result inefficient. We have proposed a more efficient Two Rate Multiplexer (TRM), which can select the output speed between two values, the nominal speed and the maximum speed, in order to satisfy the preassigned QoS requirements. The performance of TRM presented in this paper show the superiority of this approach with respect to the Peak Multiplexer and the Statistical multiplexer.

The ATM Forum has recently introduced a new class of ABR services, which require no bandwidth reservation. If no congestion control is enforced, the cell loss becomes very high during overload network conditions. The rate-based control, namely the proportional rate control algorithm (PCRA), has been proposed by ATM Forum. The PCRA is based on the concept that, in absence of an explicit notification indicating no congestion in the network, each source gradually decreases its cell rate until the permitted minimum value is reached. The simulation model considered consists of two ATM switches connected through a VP at 155 Mbit/s. Each switch is equipped with buffers at each input and output port. The main goal of our simulation has been to investigate the system performance, taking into account the influence of the various parameters. Two policies have been assumed to detect the congestion status in the switch. In the first, only the queue length is considered, while in the second, the gradient of the queue length is also taken into account, in order to react faster to an emerging congestion.

With homogeneous sources, simulation results show that a fair behavior among different sources is obtained. Variations on the transmission rate and on the queue length are present during the system operation. These variations are drastically reduced when the gradient policy is used. Moreover, the ICR value also influences the system performance, producing higher variations on the queue length, if it is set at higher values. These preliminary results confirm the need to increase the buffer length in ATM switches (at present, ATM switches are equipped with buffer of less 100 cells) if $\mathrm{ABR}$ services must be provided. In this context, it is also advisable to choose small values of ICR.

With heterogeneous sources simulation results show that the performance characteristics are more complicated to manage. The faster source is subject to strong variations of transmission rate and buffer length. The same behavior is observed for a source connected to the switch through a long link (100 km as opposed to $10 \mathrm{~km}$, in our example).

In a multiswitch network, not considered in this paper, the effect of different propagation delays is expected to be even more critical. Further studies are in progress to investigate the possibility to adopt a policy in the ATM switch for handling the parameter ER. Improvements are expected but at the cost of more complex functions to be performed by ATM switches. The trade off will be carefully studied. 


\section{REFERENCES}

[1] Lee, B.G., Kang, M. and Lee, J. (1993) Broadband telecommunications technology. Artech House. Boston-London.

[2] Iwata, A., Mori, N., Ikeda, C., Suzuki, H. and Ott, M. (1995) ATM connection and traffic management schemes for multimedia internetworking. Communications of ACM. February 1995, 38, Number 2.

[3] Fratta, L., Musumeci, L., Passalia, A. and Rigolio, G. (1993) Performance of frame relay services on ATM networks. Proceedings of the Fifth International Conference on Data Communication Systems and their Performance. Raleigh, North Carolina, USA. October, 1993.

[4] Fratta, L., Gallassi, G., Musumeci, L. and Verri, L. (1992) Congestion control strategies in ATM networks. ETT, 3, 2, Mar.-Apr. 1992, pp. 183-193.

[5] CCITT, Recommendation I. 364: Support of broadband connectionless data services on B-ISDN. Study Group XVIII, Geneve, June 1992.

[6] Cappellini, G., Decina, M., Fratta, L. and Musumeci, L. (1993) SMDS variable rate access in ATM networks. Globecom '93. Houston, Texas. Dec. 1993.

[7] Bonomi, F. and Findick, K.W. (1995) The rate-based flow control framework for the available bit rate ATM service. IEEE Network. March/April 1995.

[8] Siu, K.and Tzeng, H. (1995) Intelligent congestion control for ABR service in ATM networks. Computer Communication Review. April 1995.

[9] ATM Forum/94-0974. New pseudocode for explicit rate plus EFCI support. October 1994.

[10] Kolarof, A. and Ramamurthy, G. (1994) Comparison of congestion control schemes for ABR services in ATM local area network. Globecom '94. San Francisco, California. Dec. 1994.

[11] Pozzi, D. (1995) "Preliminary results on ABR Service". DEI Internal report, June 1995.

Luigi Fratta received the Doctorate in electrical engineering from the Politecnico di Milano, Milano, Italy, in 1966. From 1967 to 1970 he worked at the Laboratory of Electrical Communications, Politecnico di Milano. As a Research Assistant at the Department of Computer Science, University of California, Los Angeles, he participated in data network design under the ARPA project from 1970 to 1971. From November 1975 to September 1976 he was at the Computer Science Department of the IBM Thomas J. Watson Research Center, Yorktown Heights, NY, working on modeling analysis and optimization techniques for teleprocessing systems. In 1979 he was a Visiting Associate Professor in the Department of Computer Science at the University of Hawaii. In the summer of 1981 he was at the Computer Science Department, IBM Research Center, San José, CA, working on local area networks. During the summers of 1983 and 1989 he was with the Research in Distributed Processing Group, Department of Computer Science, U.C.L.A., working on fiber optic local area networks. During the summer of 1986 he was with Bell Communication Research working on metropolitan area networks. At present he is a Full Professor at the Dipartimento di Elettronica e Informazione of the Politecnico di Milano. His current research interests include computer communication networks, packet witching networks, multiple access systems, modeling and performance evaluation of communication systems, and local area networks.

Dr. Fratta is a member of the Association for Computing Machinery and the Italian Electrotechnical and Electronic Association. 
Luigi Musumeci is an Associate Professor at the Electronic and Information Department of the Politecnico di Milano.

He received his degree in Electrical Engineering from the Politecnico di Milano in 1961.

From 1968 to 1986, he was at Italtel, where he was responsible for the design and implementation of ITAPAC the Italian packet network.

His research interests cover packet data networks, wireless networks, and B-ISDN. He is the author of over 40 articles on these topics. 